\title{
Physicochemical Analysis of Water Quality of Brook Kuruçay
}

\author{
Ekrem Mutlü, Arzu Aydın Uncumusaoğlu ${ }^{2} *$ \\ ${ }^{1}$ Departments of Aquaculture, Faculty of Fisheries, Kastamonu University, 37150 Kastamonu, Turkey \\ ${ }^{2}$ Departments of Environmental Engineering, Faculty of Engineering, Giresun University, 28200 Giresun, Turkey
}

\section{A R T I C L E I N F O}

Article history:

Received 09 August 2016

Accepted 28 September 2016

Available online, ISSN: 2148-127X

Keywords:

Water Pollution

Water Quality

Surface water

Hafik

Brook Kuruçay

* Corresponding Author:

E-mail: arzu.a.uncumusaoglu@gmail.com

\section{A B S T R A C T}

In this study, through the analyses of water samples taken from 9 stations on the brook between July 2012 and June 2013, we aimed to determine the monthly and seasonal changes in water quality parameters of Brook Kuruçay, to determine the water quality properties, to reveal the pollution problems, to determine the suitability level in terms of aquatic life and to classify the quality of water in accordance with Surface Water Quality Management Regulation's Inland Surface Water Classes criteria. The study area is located southeast of the Hafik District of Sivas city and the altitude is $2608 \mathrm{~m}$. The water samples were collected from 9 stations established on the brook, and some physicochemical parameters and heavy metal concentrations were analyzed in water samples. The cleaning and maintenance of all of the equipment, land-type measurement tools, and glass sampling containers to be used in sampling were made 1 day before sampling. Sampling tubes were immersed into $15 \mathrm{~cm}$ below the water surface for taking water samples. Heavy metal concentrations were determined in the Sivas Provincial Control Laboratory in the same day with sampling (within 5 hours). The total alkalinity, total hardness, ammonium nitrogen, nitrite, nitrate, ammonium azote, phosphate, sulfite, sulfate, chloride, sodium, potassium, suspended solid matter (SSM), chemical oxygen demand (COD), biological oxygen demand (BOD), calcium, magnesium, ferrous, lead, copper, zinc, nickel, mercury and cadmium analyses of water samples were performed.

As a result of the analyses, it was determined that, since Brook Kuruçay falls into the water resource class, which is the most sensitive to pollution, the water quality of the brook should be monitored regularly.

\section{Kuruçay Deresinin Su Kalitesinin Fiziko-Kimyasal Analizi}

\section{A K A LE B İ L G İ İ}

Geliş 09 Ağustos 2016

Kabul 28 Eylül 2016

Çevrimiçi baskı, ISSN: 2148-127X

Anahtar Kelimeler:

Su Kirliliği

Su Kalitesi

Yüzey suyu

Hafik

Kuruçay Deresi

${ }^{*}$ Sorumlu Yazar:

E-mail: arzu.a.uncumusaoglu@gmail.com

\section{Ö Z E T}

Bu çalışmada, Temmuz 2011 ile Haziran 2012 arasında 9 istasyondan alınan su örneklerinin analizi ile Kuruçay deresinin su kalitesi parametrelerindeki ayllk ve mevsimsel değişimleri incelemek, su kalitesi özelliklerini belirlemek, kirlilik problemlerini ortaya çıkarmak, sucul yaşam işin uygunluğu belirlemek ve Yüzey Suları Kalite Kontrol Yönetmeliğinin Kıta İçi Yüzey Suları Sınıflandırması kriterlerine göre su kalitesini sınıflandırması amaçlanmıştır. Çalışma alanı Sivas ili Hafik ilçesinin güneydoğu kesiminde yer almakta ve rakım 2608m'dir. Dere üzerindeki 9 istasyondan su örnekleri alınmış ve bu su örneklerinde bazı fiziko -kimyasal parametreler ve ağır metal konsantrasyonları analiz edilmiştir. Ekipmanların, saha tipi ölçüm aletlerinin ve cam örnekleme kaplarının temizlik ve bakımları su örneklerini almaya çıkmadan bir gün önce gerçekleştirilmiştir. Örnekleme tüpleri su yüzeyinin $15 \mathrm{~cm}$ altına daldırılmış ve suyun kendi cazibesi ile tüpe dolması sağlanmıştır. Ağır metal konsantrasyonları Sivas İl Kontrol Laboratuvarı'nda aynı gün, en geç 5 saat içerisinde gerçekleştirilmiştir. Toplam alkalinite, toplam sertlik, amonyum azotu, fosfat, nitrit, nitrat, sülfat, sodyum, potasyum, askıda katı madde (AKM), kimyasal oksijen ihtiyacı (KOİ), biyolojik oksijen ihtiyac1 (BOI), kalsiyum, magnezyum, demir, kurşun, bakır, çinko, nikel, civa ve kadmiyum analizleri gerçekleştirilmiştir. Analizler sonucunda; Kuruçay deresi, çevresindeki bazı köylerin içme suyu ihtiyacını da karşıladığı için kirliliğe karşı en yüksek düzeyde hassas su kaynağı sınıfına girmektedir. Bu sebeple Kuruçay deresinin su kalitesi düzenli olarak izlenmelidir. 


\section{Introduction}

Water is a very important material having vital importance for human and other organisms. It is used for drinking, cleaning, domestic and agricultural purposes (Kaptan and Özan, 2014). Throughout the history, people have preferred the sites around the sources, where it has been easy to access the water, and the river sides as residential areas (Alaş and Çil, 2002).

Among the total water stock amount, the portion of lakes and rivers in directly-usable form is only $0.27 \%$ (Gleick, 1996). Even though this is a very low rate, the organisms have to use this limited amount of fresh water sources in order to meet their water-related necessities (Tüzun et al., 2006).

The rivers are the ecosystems, which are affected by environmental pollution at most. The release of domestic, industrial-, and agricultural-origin pollutants into rivers without adequate filtering and their accumulation lead to water pollution, and many organisms living in streams face with the risk of extinction. Such events obviously indicate the necessity of researching the pollution level of streams (Taş, 2006).

Most of surface water sources are the areas, where the industrial, agricultural and domestic wastes are discharged in countries such as ours, they are also the sources used in meeting potable water, usage water, irrigation water and aquaculture capital needs. In determining the use of these sources, it is very important to know the physical, chemical, and biological properties of the water source. For this purpose, many studies are carried out in our country; Akkan et al., 2011; Kurnaz et al., 2016; Polat and Akkan, 2016).

Because of the release of domestic wastewaters and sewages into the river from Uzunbelen, Dündar, Kolköy and Değirmen villages, and the leakage of fertilizers and pesticides from the near agricultural lands, Brook Kuruçay, where this study was carried out, is under the risk of pollution.

In this study on Brook Kuruçay, it was aimed to determine the monthly and seasonal changes in water quality parameters of Brook Kuruçay, to determine the water quality properties, to reveal the pollution problems, to determine the suitability level in terms of aquatic life, and to classify the quality of water in accordance with Surface Water Quality Management Regulation's Inland Surface Water Classes criteria.

\section{Materials and Methods}

The study area is located southeast of Hafik District of Sivas city. The altitude is $2608 \mathrm{~m}$. Brook Kuruçay emerges from Mount Gürlevik and merges with Kızılırmak within the borders of the Tavşanlı Village of Hafik district.

The locations of sampling stations are as follows; $1^{\text {st }}$ station: resource of Brook Kuruçay (Aktaş Village), $2^{\text {nd }}$ station: excitation from Aktaş Village, $3^{\text {rd }}$ station: entrance of Kabalı Village, $4^{\text {th }}$ station: excitation from Kabalı village entrance, $5^{\text {th }}$ station: entrance into Süleymaniye village, $6^{\text {th }}$ station: excitation from Süleymaniye village, $7^{\text {th }}$ station: entrance into Bakıml village, $8^{\text {th }}$ station: excitation from Bakımlı Village, last station: point of merging with Yeşilırmak within the borders of Değirmene Village (within the borders of Almus district) (Figure 1).

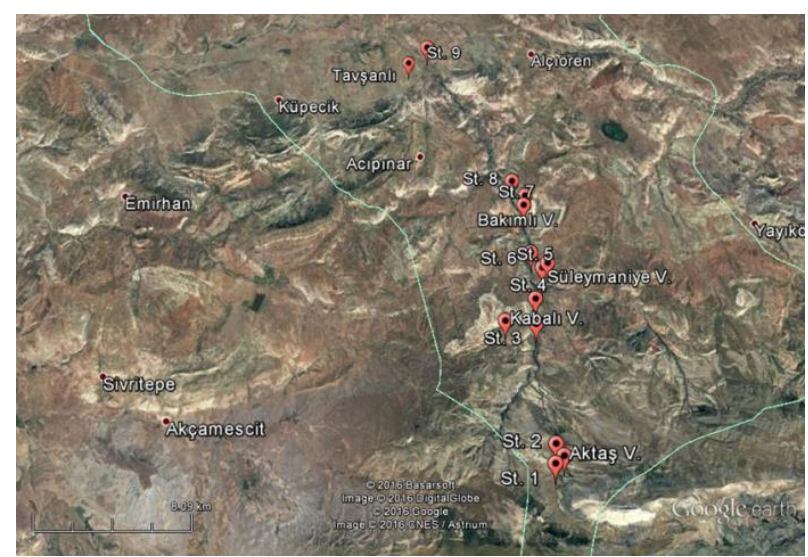

Figure 1 Map of study area with sampling point locations

\section{Water Analysis}

In this study starting from July 2011, samples used in analyses of chemical and physical parameters constituting the water quality were monthly collected from 9 stations for 12 months. The sampling ended at June 2012. The sampling tubes to be used in water sampling were flushed and immersed into $15 \mathrm{~cm}$ below water surface for taking water samples.

The obtained water samples were taken to the laboratory within maximum 5 hours for analysis. Temperature, $\mathrm{pH}$, electrical conductivity and dissolved oxygen, parameters were measured in-place via land-type measurement devices. Temperature and dissolved oxygen were measured via YSI brand S2 model oxygen-meter, $\mathrm{pH}$ measurement was conducted with Orion brand 420A model pH-meter, and saltiness (ppt) and the electrical conductance $(\mu \mathrm{s} / \mathrm{cm})$ were measured by using YSI brand 30/50 FT model conductance-meter.

Among other parameters determining water quality; total alkalinity, total hardness, ammonium nitrogen, nitrite, nitrate, phosphate, sulfite, sulfate chloride, sodium, potassium, suspended solid matter (SSM), chemical oxygen demand (COD), biological oxygen demand (BOD), calcium, magnesium, ferrous, lead, copper, zinc, nickel, mercury and cadmium analyses of water samples were conducted in the laboratory of Sivas Directorate of Provincial Food Agriculture and Livestock in the same day.

Titration with sulfuric acid (for total alkalinity) and titration with EDTA (for total hardness) were executed. The results were presented in $\mathrm{mg} / \mathrm{L} \mathrm{CaCO}_{3}$ unit. Chemical oxygen level was calculated through titration with ferrous ammonium sulfate based on determination of amount of oxygen being used while lysing the natural and organic pollutant load by using powerful chemical oxidants. The level of biological oxygen was calculated via WTW brand Oxi Top BSB BOD DBO biological oxygen measurement device. The analyses of ammoniac, nitrite, nitrate, ammonium nitrogen $\left(\mathrm{NH}_{4}^{+}\right)$, phosphate, sulfate, sulfite, chloride, sodium, potassium, calcium, and magnesium were conducted with Hack Lange's DR3900 desktop spectrophotometer by using Merck photometric test kits according to standard methods for the Examination of 
Water and Wastewater (Anonymous, 1998). The analyses of lead, copper, ferrous and cadmium, mercury, nickel, and zinc of water samples were conducted with Perkin Elmer's Optima 2000 DV ICP-OES device in laboratory. The analysis of Suspended Solid Matter (SSM) was conducted by filtering the water through Whatman brand $42 \mathrm{Nr} 0.45 \mathrm{NM}$ membrane filters, and then keeping filter papers at $103^{\circ} \mathrm{C}$ for 24 hours and calculating the weight difference. Annual mean values, seasonal mean values and stations' mean values of each of the parameters were calculated by using Office Excel 2007, which is a part of Microsoft Office Professional Edition 2007. Descriptive statistical analysis including One-way ANOVA, significance (0.05) was done. Important differences in the mean values were tested with Tukey's multiple range test. All statistical calculations were performed with SPSS 17.0 for Windows.

\section{Results and Discussion}

The annual mean values of the parameters by the stations are presented in Table $1 \mathrm{a}, \mathrm{b}$, while the seasonal mean values are presented in Table 2. There were significant differences (ANOVA, $\mathrm{P}>0.05$ ) in average surface temperatures among stations and seasons (Table 1a,ba,b; Table 2). One of the most important factors that affect the biological activity of the aquatic organisms and fish is the water temperature. The changes in this parameter result from seasonal temperature changes (Mutlu et al., 2013c). Brook Kuruçay shows inland water characteristic. The temperature differences measured in 9 stations during the study were not at the level that can affect the aquatic life negatively (Table 1a,ba,b).

$\mathrm{pH}$ is another parameter that indicates the chemical and biological properties. It is used for classifying the weak acid and bases. This separation affects the toxicity of many compounds (Atay and Pulatsü, 2000). In order for a $\mathrm{pH}$ value of any aquatic medium to not threaten the aquatic life and in order for a water resource to be suitable for aquaculture, it should fit within the range of $6.5-8.5$ (Kara and Gömlekçioğlu, 2004). The mean value of water samples taken from Brook Kuruçay during the year was found to be 7.56, while the maximum mean value was 7.68 in $8^{\text {th }}$ station. According to these results, the brook has mildly basic character, and is first class in accordance with RSWQM in terms of $\mathrm{pH}$ value, and suitable for aquaculture.

Another parameter influencing the development of a balanced fauna is the dissolved oxygen (DO) concentration. Besides being a compound that is necessary for aquatic life, DO is also necessary for biochemical oxidations. In sweet waters, there should be at least $5 \mathrm{mg} / \mathrm{L}$ dissolved oxygen for aquatic life (Atay and Pulatsü, 2000). The lowest mean DO value measured in this study was found to be $8.63 \mathrm{mg} / \mathrm{L}$ in $9^{\text {th }}$ station, so the water of Brook Kuruçay is suitable for aquaculture in terms of DO concentration, and it is in Class I in accordance with RSWQM (Table 1a,b).

Chemical Oxygen Demand (COD) is another parameter that is utilized in order to find the pollution level of waters and waste waters (Mutlu et al., 2013a). The COD level of waters higher than $25 \mathrm{mg} / \mathrm{L}$ indicate the pollution, while the values more than $50 \mathrm{mg} / \mathrm{L}$ indicates the severe pollution and possible toxicity for aquatic animals (Güler, 1997). The maximum mean COD value measured in Brook Kuruçay was determined to be 1.29 $\mathrm{mg} / \mathrm{L}$ in $9^{\text {th }}$ station. According to the RSWQM and the rule that the worst value determines the class, the Brook Kuruçay is in Class I in terms of COD (Table 1a,b).

Biological Oxygen Demand (BOD) shows the amount of oxygen required by the microorganisms for dissolving the organic matters in an aquatic environment under aerobic conditions. It is utilized in order to determine an environment's pollution potential and a receiver environment's assimilation capacity by calculating the amount of dissolved oxygen that they consume while being released into the receiving mediums. The maximum mean BOD value in Brook Kuruçay was found to be 0.78 $\mathrm{mg} / \mathrm{L}$ in $9^{\text {th }}$ station, and it is Class I according to RSWQM in terms of BOD (Table 1a,b).

Electrical conductivity (EC) is very important for aquatic products, and the conductivity passes beyond the level of $100 \mu \mathrm{s} / \mathrm{cm}$ as the pollution increases (Verep et al., 2005). The electrical conductivity values have decreased in winter months, and increased in months when the water temperature and inorganic salts in the system increased. The maximum mean EC value measured in the brook was measured to be $221.72 \mu \mathrm{s} / \mathrm{cm}$ in $9^{\text {th }}$ station (Table 1a,b).

Suspended Solid Matter (SSM) amount consists of inorganic matters such as clay and loam. The maximum acceptable level of SSM in aquaculture was specified as $10 \mathrm{mg} / \mathrm{L}$ (Ntenque, 2006). The maximum mean SSM amount determined in Brook Kuruçay during the year was found to be $3.25 \mathrm{mg} / \mathrm{L}$ in $9^{\text {th }}$ station, which means that the conditions in the brook is suitable for aquaculture activities (Table 1a,b).

The sources of the nitrogen mixing into surface waters can be originated from natural domestic and agricultural resources (Mutlu et al., 2014). The nitrite $\left(\mathrm{NO}_{2}\right)$ sources in waters are the organic matters, nitrogenous fertilizers, and some of minerals. The nitrite concentration in waters higher than $1 \mathrm{mg} / \mathrm{L}$ indicates pollution (Taş, 2011). While the concentration of $\mathrm{NO}_{2}$ in natural waters is low, it is high in waters where the organic pollution is high (İmamoğlu, 2000). Nitrogen derivatives of nitrite $\left(\mathrm{NO}_{2}\right)$, nitrate $\left(\mathrm{NO}_{3}\right)$ and ammonium nitrogen $\left(\mathrm{NH}_{4}{ }^{+}\right)$have significant roles in water pollution, and they also have significant effects on the level of dissolved oxygen and eutrophication. According to the RSWQM, the brook shows Class I water characteristic in terms of nitrite $\left(\mathrm{NO}_{2}\right)$ and ammonium nitrogen (Table 1a,b).

Nitrate $\left(\mathrm{NO}_{3}\right)$ is the final product of nitrogenous organic matters. High concentration of nitrate in surface waters indicates that the water was polluted before by the industrial and domestic wastewaters containing ammonium and organic nitrogen and the fertilizers used in agricultural lands and containing nitrate (Topal and Arslan Topal, 2012). Even though the low doses of nitrate are not toxic for fish, it was reported that fish mortality starts at the doses of $4 \mu \mathrm{g} / \mathrm{L}$ and higher (Acu, 2000). Its concentration within surface waters is an indicator of the pollution of those waters caused by domestic and industrial waste waters containing ammonium and organic azote and the nitrogenous fertilizers used in agricultural lands (Topal and Arslan Topal, 2012). According to the RSWQM, Brook Kuruçay is Class I water in terms of nitrate $\left(\mathrm{NO}_{3}\right)$. 
Table 1a Stations Mean, Standard deviation (SD) and range (Minimum- maximum) of water quality parameters

\begin{tabular}{|c|c|c|c|c|c|}
\hline Parameters & St. 1 & St. 2 & St. 3 & St. 4 & St. 5 \\
\hline \multirow{2}{*}{$\mathrm{DO}(\mathrm{mg} / \mathrm{L})$} & $12.23 \pm 0.73^{d}$ & $11.95 \pm 0.85^{\mathrm{d}}$ & $11.73 \pm 0.89^{\mathrm{cd}}$ & $11.57 \pm 0.94^{\mathrm{cd}}$ & $11.45 \pm 1.12^{\mathrm{cd}}$ \\
\hline & $11.20-13.38$ & $10.68-13.22$ & $10.32-12.96$ & $10.08-12.74$ & $9.64-12.86$ \\
\hline \multirow{2}{*}{ Salinity } & $0.0183 \pm 0.006^{\mathrm{a}}$ & $0.0325 \pm 0.01^{\mathrm{ab}}$ & $0.0358 \pm 0.01^{\mathrm{abc}}$ & $0.0425 \pm 0.015^{\mathrm{bcd}}$ & $0.0483 \pm 0.018^{\mathrm{bcd}}$ \\
\hline & $0.010-0.030$ & $0.020-0.050$ & $0.020-.060$ & $0.020-0.070$ & $0.020-0.080$ \\
\hline \multirow{2}{*}{$\mathrm{pH}$} & $7.556 \pm 0.097^{\mathrm{ab}}$ & $7.477 \pm 0.11^{\mathrm{a}}$ & $7.503 \pm 0.121^{\mathrm{ab}}$ & $7.540 \pm 0.138^{\mathrm{ab}}$ & $7.570 \pm 0.152^{\mathrm{ab}}$ \\
\hline & $7.42-7.70$ & $7.320-7.660$ & $7.340-7.700$ & $7.350-7.750$ & $7.370-7.820$ \\
\hline \multirow{2}{*}{$\begin{array}{l}\text { Temp. } \\
\left(\mathrm{C}^{\circ}\right)\end{array}$} & $9.2083 \pm 1.44$ & $11.991 \pm 4.87$ & $12.216 \pm 5.29$ & $12.733 \pm 6.05$ & $13.133 \pm 6.68$ \\
\hline & $7.50-11.30$ & $6.60-18.70$ & $6.40-19.80$ & $6.20-22.10$ & $6.00-24.20$ \\
\hline \multirow{2}{*}{$\mathrm{EC}(\mu \mathrm{s} / \mathrm{cm})$} & $152.41 \pm 10.67^{\mathrm{a}}$ & $176.31 \pm 14.71^{\mathrm{ab}}$ & $180.10 \pm 18.23^{\mathrm{ab}}$ & $183.31 \pm 24.56^{\mathrm{b}}$ & $190.26 \pm 25.61^{b}$ \\
\hline & 136.94-168.28 & $157.52-199.18$ & $157.59-208.40$ & $140.28-218.30$ & $159.30-230.00$ \\
\hline S.S.M. & $0.2867 \pm 0.216^{\mathrm{a}}$ & $1.4783 \pm 0.626^{\mathrm{ab}}$ & $1.723 \pm 0.760^{\mathrm{bc}}$ & $2.127 \pm 0.962^{\mathrm{bcd}}$ & $2.4467 \pm 1.083^{\mathrm{bcd}}$ \\
\hline$(\mathrm{mg} / \mathrm{L})$ & 0.06-0.90 & $0.620-2.52$ & $0.720-2.920$ & $0.820-3.820$ & $0.920-4.220$ \\
\hline C.O.D. & $0.1225 \pm 0.023^{\mathrm{a}}$ & $0.6517 \pm 0.354^{\mathrm{ab}}$ & $0.854 \pm 0.517^{\mathrm{b}}$ & $0.992 \pm 0.602^{b}$ & $1.0933 \pm 0.648^{\mathrm{b}}$ \\
\hline$(\mathrm{mg} / \mathrm{L})$ & $0.070-0.150$ & $0.200-1.240$ & $0.230-1.900$ & $0.260-2.140$ & $0.280-2.220$ \\
\hline B.O.D. & $0.0808 \pm 0.017^{\mathrm{a}}$ & $0.345 \pm 0.207^{\mathrm{ab}}$ & $0.464 \pm 0.269^{a b c}$ & $0.574 \pm 0.317^{\mathrm{bc}}$ & $0.653 \pm 0.367^{\mathrm{bc}}$ \\
\hline$(\mathrm{mg} / \mathrm{L})$ & $0.05-0.110$ & $0.160-0.840$ & $0.170-1.000$ & $0.190-1.100$ & $0.20-1.220$ \\
\hline \multirow{2}{*}{$\mathrm{Cl}^{-}(\mathrm{mg} / \mathrm{L})$} & $7.589 \pm 0.721^{\mathrm{a}}$ & $7.323 \pm 1.275^{\mathrm{a}}$ & $7.282 \pm 1.281^{\mathrm{a}}$ & $7.218 \pm 1.335^{\mathrm{a}}$ & $7.177 \pm 1.360^{\mathrm{a}}$ \\
\hline & $5.340-8.020$ & $4.12-8.34$ & $4.08-8.29$ & $3.90-8.27$ & $3.82-8.25$ \\
\hline \multirow{2}{*}{$\mathrm{PO}_{4}(\mathrm{mg} / \mathrm{L})$} & $0.0036 \pm 0.001^{\mathrm{a}}$ & $0.0080 \pm 0.004^{b c}$ & $0.0091 \pm 0.006^{\mathrm{bc}}$ & $0.0120 \pm 0.008^{\mathrm{abc}}$ & $0.015 \pm 0.012^{\mathrm{abcd}}$ \\
\hline & $0.002-0.006$ & $0.004-0.017$ & $0.0007-0.023$ & $0.004-0.0320$ & $0.004-0.0440$ \\
\hline $\mathrm{SO} 4^{2-}$ & $2.631 \pm 0.84^{\mathrm{a}}$ & $18.743 \pm 12.40^{\mathrm{bc}}$ & $23.33 \pm 14.75^{b c}$ & $31.087 \pm 20.24^{\mathrm{bc}}$ & $39.335 \pm 26.95^{\mathrm{b}}$ \\
\hline$(\mathrm{mg} / \mathrm{L})$ & $1.740-4.220$ & $2.840-39.140$ & $4.040-45.300$ & $4.520-56.110$ & $4.58-70.960$ \\
\hline \multirow{2}{*}{$\mathrm{S}_{2}{ }^{-}(\mathrm{mg} / \mathrm{L})$} & $0.4883 \pm 0.306^{\mathrm{a}}$ & $1.0917 \pm 0.596^{\mathrm{ab}}$ & $1.3167 \pm 0.67^{\mathrm{abc}}$ & $1.6000 \pm 0.822^{\mathrm{bcd}}$ & $1.8667 \pm 0.929^{\mathrm{bcd}}$ \\
\hline & $0.130-1.1300$ & $0.250-2.1500$ & $0.350-2.4500$ & $0.450-3.2500$ & $0.550-3.5500$ \\
\hline \multirow{2}{*}{$\mathrm{Na}(\mathrm{mg} / \mathrm{L})$} & $18.96 \pm 0.328^{\mathrm{a}}$ & $29.755 \pm 4.71^{\mathrm{ab}}$ & $30.14 \pm 5.48^{\mathrm{ab}}$ & $32.013 \pm 6.93^{\mathrm{b}}$ & $38.511 \pm 19.96^{\mathrm{b}}$ \\
\hline & $18.08-19.330$ & $22.66-37.88$ & $22.68-40.10$ & $23.59-44.79$ & $24.71-96.80$ \\
\hline \multirow{2}{*}{$\mathrm{K}(\mathrm{mg} / \mathrm{L})$} & $2.648 \pm 0.116^{\mathrm{a}}$ & $3.299 \pm 0.231^{\mathrm{b}}$ & $3.331 \pm 0.241^{\mathrm{b}}$ & $3.444 \pm 0.329^{b}$ & $3.518 \pm 0.374^{\mathrm{bc}}$ \\
\hline & $2.450-0.006$ & $2.890-0.017$ & $2.910-0.023$ & $2.930-0.032$ & $2.940-0.044$ \\
\hline \multirow{4}{*}{$\begin{array}{l}\text { T.Hard. } \\
\text { (mg/L) } \\
\text { T.Alka. } \\
\text { (mg/L) }\end{array}$} & $130.35 \pm 5.28^{\mathrm{a}}$ & $139.37 \pm 10.38^{\mathrm{ab}}$ & $140.36 \pm 11.18^{\mathrm{ab}}$ & $143.28 \pm 14.20^{\mathrm{ab}}$ & $144.46 \pm 16.16^{\mathrm{ab}}$ \\
\hline & $123.7-141.78$ & $125.62-158.58$ & $125.64-161.57$ & $125.84-172.34$ & $125.9-175.64$ \\
\hline & $134.44 \pm 5.18$ & $142.27 \pm 10.39$ & $143.36 \pm 11.23$ & $146.28 \pm 14.32$ & $148.30 \pm 15.56$ \\
\hline & $127.79-145.66$ & $128.5-161.30$ & $128.52-164.72$ & $128.58-175.82$ & $128.66-179.00$ \\
\hline \multirow{2}{*}{$\operatorname{Mg}(\mathrm{mg} / \mathrm{L})$} & $7.3317 \pm 0.089$ & $7.5583 \pm 0.155$ & $7.5825 \pm 0.168$ & $7.6633 \pm 0.287$ & $7.550 \pm 0.718$ \\
\hline & $7.200-7.520$ & $7.380-7.900$ & $7.390-7.940$ & $7.420-8.440$ & $5.530-8.600$ \\
\hline \multirow{2}{*}{$\mathrm{Ca}(\mathrm{mg} / \mathrm{L})$} & $13.37 \pm 19.82$ & $7.88 \pm 0.228$ & $7.92 \pm 0.263$ & $8.00 \pm 0.354$ & $8.09 \pm 0.452$ \\
\hline & $7.430-76.300$ & $7.610-8.390$ & $7.620-8.470$ & $7.650-8.830$ & $7.670-9.210$ \\
\hline \multirow{2}{*}{$\mathrm{NO}_{2}(\mathrm{mg} / \mathrm{L})$} & $0.0003 \pm 0.000^{\mathrm{a}}$ & $0.0007 \pm 0.000^{\mathrm{ab}}$ & $0.0008 \pm 0.00^{\mathrm{abc}}$ & $0.0009 \pm 0.000^{\mathrm{bc}}$ & $0.0010 \pm 0.00^{\mathrm{bcd}}$ \\
\hline & $0.0001-0.0007$ & $0.0003-0.0012$ & $0.0003-0.0014$ & $0.0003-0.0016$ & $0.0003-0.0018$ \\
\hline \multirow{2}{*}{$\mathrm{NO}_{3}(\mathrm{mg} / \mathrm{L})$} & $0.625 \pm 0.382^{\mathrm{a}}$ & $1.083 \pm 0.570^{\mathrm{ab}}$ & $1.275 \pm 0.69^{\mathrm{abc}}$ & $1.517 \pm 0.807^{\mathrm{bc}}$ & $1.783 \pm 1.012^{\mathrm{bc}}$ \\
\hline & $0.200-1.300$ & $0.400-2.100$ & $0.400-2.600$ & $0.500-3.000$ & $0.500-3.700$ \\
\hline \multirow{2}{*}{$\begin{array}{l}\mathrm{NH}_{3}-\mathrm{N} \\
(\mathrm{mg} / \mathrm{L})\end{array}$} & $0.0003 \pm 0.000^{\mathrm{a}}$ & $0.0008 \pm 0.00^{\mathrm{ab}}$ & $0.0009 \pm 0.00^{\mathrm{bc}}$ & $0.0010 \pm 0.00^{\mathrm{bc}}$ & $0.0011 \pm 0.001^{\mathrm{bc}}$ \\
\hline & $0.0001-0.0008$ & $0.0004-0.0015$ & $0.0004-0.0017$ & $0.0004-0.0018$ & $0.0003-0.0020$ \\
\hline \multirow{2}{*}{$\mathrm{Fe}_{2}^{+}(\mathrm{mg} / \mathrm{L})$} & $0.0011 \pm 0.000^{\mathrm{a}}$ & $0.0038 \pm 0.00^{\mathrm{ab}}$ & $0.004 \pm 0.002^{\mathrm{abc}}$ & $0.0053 \pm 0.003^{\mathrm{bc}}$ & $0.0058 \pm 0.003^{b c}$ \\
\hline & $0.0100-0.100$ & $0.500-1.000$ & $0.500-1.200$ & $0.500-1.300$ & $0.600-1.500$ \\
\hline \multirow{2}{*}{$\mathrm{Fe}(\mu \mathrm{g} / \mathrm{L})$} & $0.0925 \pm 0.026^{\mathrm{a}}$ & $0.6583 \pm 0.183^{\mathrm{b}}$ & $0.8083 \pm 0.239^{b c}$ & $0.8833 \pm 0.252^{\mathrm{bcd}}$ & $1.008 \pm 0.278^{\mathrm{bcd}}$ \\
\hline & $0.0100-0.1000$ & $0.5000-1.0000$ & $0.500-1.2000$ & $0.5000-1.3000$ & $0.6000-1.5000$ \\
\hline & $1.583 \pm 1.084^{\mathrm{a}}$ & $9.417 \pm 2.843^{\mathrm{b}}$ & $10.917 \pm 2.968^{\mathrm{bc}}$ & $12.750 \pm 3.671^{\mathrm{bcd}}$ & $14.167 \pm 4.174^{\mathrm{bcd}}$ \\
\hline $\mathrm{Cu}(\mu \mathrm{g} / \mathrm{L})$ & $1.000-4.000$ & $7.000-16.0000$ & $7.000-17.0000$ & $7.000-20.0000$ & $8.000-23.0000$ \\
\hline (I & $0.0000 \pm 0.000^{\mathrm{a}}$ & $0.2000 \pm 0.135^{\mathrm{ab}}$ & $0.275 \pm 0.196^{\mathrm{abc}}$ & $0.375 \pm 0.245^{\mathrm{abcd}}$ & $0.483 \pm 0.341^{\text {bcde }}$ \\
\hline $\mathrm{Cd}(\mu \mathrm{g} / \mathrm{L})$ & $0.000-0.0000$ & $0.1000-0.5000$ & $0.1000-0.700$ & $0.100-0.9000$ & $0.100-1.200$ \\
\hline & $0.0000 \pm 0.000^{\mathrm{a}}$ & $0.0012 \pm 0.000^{\mathrm{ab}}$ & $.0019 \pm 0.001^{\mathrm{abc}}$ & $0.0031 \pm 0.002^{\mathrm{bcd}}$ & $0.0039 \pm 0.002^{\text {bcd }}$ \\
\hline $\mathrm{Hg}$ & $0.0000-0.0000$ & $0.0010-0.0020$ & $0.0010-0.0040$ & $0.0010-0.0060$ & $0.0010-0.008$ \\
\hline & $1.000 \pm 0.000^{\mathrm{a}}$ & $2.167 \pm 0.577^{\mathrm{ab}}$ & $2.667 \pm 0.888^{\mathrm{abc}}$ & $3.417 \pm 1.564^{\mathrm{bcd}}$ & $4.167 \pm 1.749^{\text {cde }}$ \\
\hline L) & $1.000-1.000$ & $2.000-4.000$ & $2.000-5.000$ & $2.000-7.000$ & $2.000-8.000$ \\
\hline & $1.167 \pm 0.577^{\mathrm{a}}$ & $4.417 \pm 2.314^{\mathrm{ab}}$ & $5.750 \pm 3.019^{\mathrm{bc}}$ & $6.750 \pm 3.049^{\mathrm{bc}}$ & $7.500 \pm 3.680^{\mathrm{bc}}$ \\
\hline g/L) & $1.000-3.000$ & $3.000-10.000$ & $3.000-12.000$ & $3.000-13.000$ & $3.000-15.000$ \\
\hline
\end{tabular}

${ }^{\mathrm{T}}$ The different letters in same column indicate significant differences $(\mathrm{P}<0.05)$ 
Table 1b Stations Mean, Standard deviation (SD) and range (Minimum- maximum) of water quality parameters

\begin{tabular}{|c|c|c|c|c|}
\hline Parameters & St. 6 & St. 7 & St. 8 & St. 9 \\
\hline \multirow{2}{*}{$\mathrm{DO}(\mathrm{mg} / \mathrm{L})$} & $10.988 \pm 1.12^{b c d}$ & $10.528 \pm 1.12^{b c}$ & $9.989 \pm 1.04^{b}$ & $8.629 \pm 1.04^{\mathrm{a}}$ \\
\hline & $9.18-12.38$ & $8.72-11.92$ & $8.2-11.07$ & $6.9-9.71$ \\
\hline \multirow{2}{*}{ Salinity } & $0.0533 \pm 0.02^{\mathrm{bcd}}$ & $0.0575 \pm 0.020^{\text {cde }}$ & $0.0617 \pm 0.022^{\mathrm{de}}$ & $0.0717 \pm 0.022^{\mathrm{e}}$ \\
\hline & $0.020-0.090$ & $0.03-0.10$ & $0.030-0.10$ & $0.04-0.110$ \\
\hline \multirow{2}{*}{$\mathrm{pH}$} & $7.606 \pm 0.15^{\mathrm{ab}}$ & $7.641 \pm 0.166^{\mathrm{ab}}$ & $7.676 \pm 0.194^{\mathrm{b}}$ & $7.463 \pm 0.188^{\mathrm{a}}$ \\
\hline & $7.390-7.840$ & $7.410-7.870$ & $7.430-8.070$ & $7.200-7.840$ \\
\hline \multirow{2}{*}{$\begin{array}{l}\text { Temp. } \\
\left(\mathrm{C}^{\circ}\right)\end{array}$} & $13.250 \pm 6.70$ & $13.333 \pm 6.68$ & $13.433 \pm 6.69$ & $14.333 \pm 6.69$ \\
\hline & $6.10-24.30$ & $6.20-24.40$ & $6.30-24.50$ & $7.20-25.40$ \\
\hline \multirow{2}{*}{$\mathrm{EC}(\mu \mathrm{s} / \mathrm{cm})$} & $192.36 \pm 25.54^{\mathrm{bc}}$ & $194.50 \pm 25.54^{\mathrm{bc}}$ & $194.56 \pm 28.55^{b c}$ & $221.72 \pm 28.55^{\mathrm{c}}$ \\
\hline & $161.44-231.70$ & $163.58-233.84$ & $147.24-235.98$ & $174.40-263.14$ \\
\hline \multirow{2}{*}{ S.S.M. (mg/L) } & $2.612 \pm 1.06^{\mathrm{bcd}}$ & $2.8000 \pm 1.090^{\mathrm{cd}}$ & $2.9717 \pm 1.096^{\mathrm{d}}$ & $3.252 \pm 1.096^{\mathrm{d}}$ \\
\hline & $1.120-4.400$ & $1.300-4.580$ & $1.480-4.760$ & $1.760-5.040$ \\
\hline \multirow{2}{*}{ C.O.D. (mg/L) } & $1.135 \pm 0.648^{\mathrm{b}}$ & $1.1733 \pm 0.648^{\mathrm{b}}$ & $1.2142 \pm 0.647^{\mathrm{b}}$ & $1.293 \pm 0.648^{\mathrm{b}}$ \\
\hline & $0.320-2.260$ & $0.360-2.300$ & $0.410-2.340$ & $0.480-2.420$ \\
\hline \multirow{2}{*}{ B.O.D. (mg/L) } & $0.673 \pm 0.367^{\mathrm{bc}}$ & $0.693 \pm 0.367^{\mathrm{bc}}$ & $0.715 \pm 0.366^{\mathrm{bc}}$ & $0.775 \pm 0.366^{\mathrm{c}}$ \\
\hline & $0.220-1.240$ & $0.240-1.260$ & $0.260-1.280$ & $0.320-1.340$ \\
\hline \multirow{2}{*}{$\mathrm{Cl}^{-}(\mathrm{mg} / \mathrm{L})$} & $7.150 \pm 1.364^{\mathrm{a}}$ & $7.290 \pm 1.580^{\mathrm{a}}$ & $7.263 \pm 1.583^{\mathrm{a}}$ & $6.170 \pm 1.762^{\mathrm{a}}$ \\
\hline & $3.78-8.21$ & $3.74-9.92$ & $3.70-9.88$ & $2.440-8.73$ \\
\hline \multirow{2}{*}{$\mathrm{PO}_{4}(\mathrm{mg} / \mathrm{L})$} & $0.019 \pm 0.013^{\mathrm{abcd}}$ & $0.0223 \pm 0.015^{\mathrm{bcd}}$ & $0.026 \pm 0.019^{\mathrm{cd}}$ & $0.029 \pm 0.019^{\mathrm{d}}$ \\
\hline & $0.006-0.0460$ & $0.008-0.053$ & $0.010-0.073$ & $0.013-0.076$ \\
\hline \multirow{2}{*}{$\mathrm{SO}^{2-}(\mathrm{mg} / \mathrm{L})$} & $41.233 \pm 26.95^{\mathrm{b}}$ & $43.123 \pm 26.95^{\mathrm{b}}$ & $45.00 \pm 26.94^{\mathrm{b}}$ & $45.24 \pm 26.90^{\mathrm{b}}$ \\
\hline & $6.47-72.85$ & $8.36-74.74$ & $10.25-76.63$ & $10.5-76.91$ \\
\hline \multirow{2}{*}{$\mathrm{S}_{2}^{-}(\mathrm{mg} / \mathrm{L})$} & $2.0850 \pm 0.928^{b c d}$ & $2.3067 \pm 0.929^{\mathrm{cd}}$ & $2.5267 \pm 0.929^{\mathrm{d}}$ & $2.5967 \pm 0.929^{\mathrm{d}}$ \\
\hline & $0.770-3.7700$ & $0.990-3.9900$ & $1.210-4.2100$ & $1.280-4.2800$ \\
\hline \multirow{2}{*}{$\mathrm{Na}(\mathrm{mg} / \mathrm{L})$} & $34.868 \pm 8.02^{\mathrm{b}}$ & $36.471 \pm 7.90^{\mathrm{b}}$ & $37.868 \pm 7.96^{\mathrm{b}}$ & $40.748 \pm 7.96^{\mathrm{b}}$ \\
\hline & $26.19-49.60$ & $27.67-51.08$ & $29.15-52.56$ & $32.03-55.44$ \\
\hline \multirow{2}{*}{$\mathrm{K}(\mathrm{mg} / \mathrm{L})$} & $3.983 \pm 0.463^{\mathrm{c}}$ & $4.461 \pm 0.573^{\mathrm{d}}$ & $5.064 \pm 0.377^{\mathrm{e}}$ & $5.543 \pm 0.387^{\mathrm{f}}$ \\
\hline & $2.960-0.046$ & $2.980-0.053$ & $4.500-0.073$ & $4.970-0.076$ \\
\hline \multirow{2}{*}{$\begin{array}{l}\text { T.Hard. } \\
\text { (mg/L) }\end{array}$} & $147.23 \pm 15.95^{\mathrm{ab}}$ & $147.59 \pm 15.756^{\mathrm{ab}}$ & $148.48 \pm 15.88^{\mathrm{b}}$ & $145.72 \pm 15.88^{\mathrm{ab}}$ \\
\hline & $125.88-176.62$ & $125.86-177.6$ & $125.84-178.58$ & $123.08-175.82$ \\
\hline \multirow{2}{*}{ T.Alka. (mg/L) } & $149.45 \pm 15.76$ & $150.83 \pm 15.84$ & $152.04 \pm 16.15$ & $150.20 \pm 16.15$ \\
\hline & $130.12-180.46$ & $131.58-181.92$ & $132.60-183.38$ & $130.76-181.54$ \\
\hline \multirow{2}{*}{$\operatorname{Mg}(\mathrm{mg} / \mathrm{L})$} & $7.653 \pm 0.720$ & $7.758 \pm 0.721$ & $7.878 \pm 0.722$ & $7.6175 \pm 0.722$ \\
\hline & $5.650-8.720$ & $5.780-8.840$ & $5.890-8.960$ & $5.630-8.700$ \\
\hline \multirow{2}{*}{$\mathrm{Ca}(\mathrm{mg} / \mathrm{L})$} & $8.34 \pm 0.452$ & $8.60 \pm 0.422$ & $8.93 \pm 0.539$ & $8.55 \pm 0.539$ \\
\hline & $7.930-9.470$ & $8.190-9.630$ & $8.450-9.980$ & $8.070-9.60$ \\
\hline $\mathrm{NO} / \mathrm{mo} / \mathrm{I})$ & $0.0011 \pm 0.00^{\mathrm{bcd}}$ & $0.0012 \pm 0.000^{\mathrm{cd}}$ & $0.0013 \pm 0.000^{\mathrm{cd}}$ & $0.0015 \pm 0.000^{\mathrm{d}}$ \\
\hline $\mathrm{NU}_{2}(\mathrm{mg} / \mathrm{L})$ & 0.0004-0.0019 & $0.0005-0.0020$ & $0.0006-0.0021$ & $0.0008-0.0023$ \\
\hline & $1.883 \pm 1.012^{\mathrm{bc}}$ & $1.983 \pm 1.012^{\mathrm{bc}}$ & $2.083 \pm 1.012^{\mathrm{bc}}$ & $2.283 \pm 1.012^{\mathrm{c}}$ \\
\hline $\mathrm{NO}_{3}(\mathrm{mg} / \mathrm{L})$ & $0.600-3.800$ & $0.700-3.900$ & $0.800-4.00$ & $1.000-4.200$ \\
\hline $\mathrm{NH}=\mathrm{N}(\mathrm{mo} / \mathrm{I})$ & $0.0012 \pm 0.001^{\mathrm{bcd}}$ & $0.0013 \pm 0.001^{\mathrm{bcd}}$ & $0.0014 \pm 0.001^{\mathrm{cd}}$ & $0.0017 \pm 0.001^{\mathrm{d}}$ \\
\hline $\mathrm{NH}_{3}-\mathrm{N}(\mathrm{n}$ & $0.0005-0.0021$ & $0.0006-0.0022$ & $0.0007-0.0023$ & $0.001-0.0026$ \\
\hline$E_{0}$ & $0.0067 \pm 0.004^{\mathrm{bc}}$ & $0.0070 \pm 0.004^{\mathrm{bcd}}$ & $0.0078 \pm 0.004^{\mathrm{cd}}$ & $0.0108 \pm 0.004^{\mathrm{d}}$ \\
\hline $\mathrm{Fe}_{2}(\mathrm{n}$ & $0.070-1.700$ & $0.7000-2.000$ & $0.700-2.200$ & $1.200-2.700$ \\
\hline $\mathrm{Fe}(\mu \mathrm{\mu} / \mathrm{I})$ & $1.056 \pm 0.444^{\mathrm{bcd}}$ & $1.225 \pm 0.416^{\mathrm{cd}}$ & $1.317 \pm 0.480^{\mathrm{d}}$ & $1.8167 \pm 0.480^{\mathrm{e}}$ \\
\hline $\mathrm{Fe}(\mu \mathrm{g} / \mathrm{L})$ & $0.0700-1.7000$ & $0.7000-2.0000$ & $0.7000-2.2000$ & $1.2000-2.7000$ \\
\hline & $15.833 \pm 4.859^{\mathrm{cd}}$ & $17.0000 \pm 5.705^{\mathrm{d}}$ & $18.166 \pm 5.670^{\mathrm{d}}$ & $25.167 \pm 5.670^{\mathrm{e}}$ \\
\hline $\mathrm{Cu}(\mu \mathrm{g} / \mathrm{L})$ & $9.000-25.000$ & $9.000-28.0000$ & $10.00-29.00$ & $17.00-36.00$ \\
\hline $\mathrm{Cd}(\mu \mathrm{g} / \mathrm{I})$ & $0.5917 \pm 0.34^{\text {bcde }}$ & $0.6667 \pm 0.389^{\text {cde }}$ & $0.733 \pm 0.408^{\mathrm{de}}$ & $0.8333 \pm 0.408^{\mathrm{e}}$ \\
\hline $\mathrm{cd}(\mu \mathrm{g} / \mathrm{L})$ & $0.200-1.300$ & $0.200-1.500$ & $0.300-1.600$ & $0.400-1.700$ \\
\hline & $0.0045 \pm 0.002^{\text {cde }}$ & $0.0053 \pm 0.003^{\mathrm{de}}$ & $0.0059 \pm 0.004^{\mathrm{de}}$ & $0.0069 \pm 0.004^{\mathrm{e}}$ \\
\hline $\mathrm{Hg}(\mu \mathrm{g} / \mathrm{L})$ & $0.0020-0.009$ & $0.0020-0.012$ & $0.0020-0.015$ & $0.0030-0.016$ \\
\hline & $4.750 \pm 1.815^{\mathrm{de}}$ & $5.250 \pm 1.765^{\mathrm{de}}$ & $5.750 \pm 1.960^{\mathrm{e}}$ & $7.750 \pm 1.960^{\mathrm{f}}$ \\
\hline $\mathrm{N} 1(\mu \mathrm{g} / \mathrm{L})$ & $2.000-8.000$ & $3.000-9.000$ & $3.000-9.000$ & $5.000-11.000$ \\
\hline & $8.667 \pm 3.651^{\mathrm{bcd}}$ & $9.250 \pm 3.888^{\mathrm{cd}}$ & $9.833 \pm 4.366^{\mathrm{cd}}$ & $12.833 \pm 4.366^{\mathrm{d}}$ \\
\hline $\mathrm{Zn}(\mu \mathrm{g} / \mathrm{L})$ & $4.000-16.000$ & $5.000-18.000$ & $5.000-20.000$ & $8.00-20.00$ \\
\hline
\end{tabular}

\footnotetext{
The different letters in same column indicate significant differences $(\mathrm{P}<0.05)$
} 
Table 2 Seasonal Mean, Standard deviation (SD) and range (Minimum- maximum) of water quality parameters

\begin{tabular}{|c|c|c|c|c|}
\hline Parameters & Winter & Spring & Summer & Autumn \\
\hline \multirow{2}{*}{$\mathrm{DO}(\mathrm{mg} / \mathrm{L})$} & $11.8785 \pm 1.1568$ & $11.4985 \pm 0.9902^{\mathrm{bc}}$ & $9.8652 \pm 1.2680^{\mathrm{a}}$ & $10.7870 \pm 1.4685^{b}$ \\
\hline & $9.22-13.38$ & $9.10-12.44$ & $6.90-11.58$ & $6.96-12.70$ \\
\hline \multirow{2}{*}{ Salinity } & $0.0296 \pm .0113^{\mathrm{a}}$ & $0.0470 \pm 0.0159^{b}$ & $0.0670 \pm 0.0248^{c}$ & $0.0437 \pm 0.0196^{\mathrm{b}}$ \\
\hline & $0.01-0.05$ & $0.02-0.08$ & $0.02-0.11$ & $0.01-0.09$ \\
\hline \multirow{2}{*}{$\mathrm{pH}$} & $7.3804 \pm .0710^{\mathrm{a}}$ & $7.5419 \pm 0.0859^{b}$ & $7.6889 \pm 0.1011^{\mathrm{c}}$ & $7.6256 \pm 0.1630^{c}$ \\
\hline & $7.20-7.48$ & 7.39-7.68 & $7.50-7.88$ & $7.38-8.07$ \\
\hline \multirow{2}{*}{ Temp. $\left(\mathrm{C}^{\circ}\right)$} & $6.8296 \pm .5362^{\mathrm{a}}$ & $12.1111 \pm 2.5290^{\mathrm{b}}$ & $19.3185 \pm 4.0839^{c}$ & $12.2444 \pm 5.9613^{\mathrm{b}}$ \\
\hline & $6.00-8.00$ & $8.20-16.40$ & $10.50-25.40$ & $6.70-22.80$ \\
\hline \multirow{2}{*}{$\mathrm{EC}(\mu \mathrm{s} / \mathrm{cm})$} & $166.333 \pm 14.8409^{\mathrm{a}}$ & $185.2244 \pm 19.5143^{b}$ & $214.4696 \pm 25.1366^{c}$ & $183.0933 \pm 28.912^{b}$ \\
\hline & $136.94-202.48$ & $149.02-234.04$ & $160.96-263.14$ & $140.28-253.80$ \\
\hline \multirow{2}{*}{ S.S.M. (mg/L) } & $1.3022 \pm .5986^{\mathrm{a}}$ & $2.1385 \pm 0.9573^{b}$ & $3.2189 \pm 1.3103^{c}$ & $2.0944 \pm 1.2188^{\mathrm{b}}$ \\
\hline & $0.06-2.54$ & $0.21-3.74$ & $0.31-5.04$ & $0.11-4.560$ \\
\hline \multirow{2}{*}{ C.O.D. (mg/L) } & $0.3967 \pm .1973^{\mathrm{a}}$ & $0.8052 \pm 0.3111^{\mathrm{b}}$ & $1.5189 \pm 0.7038^{c}$ & $1.0700 \pm 0.6188^{\mathrm{b}}$ \\
\hline & $0.07-0.82$ & $0.12-1.24$ & $0.13-2.42$ & $0.11-2.180$ \\
\hline \multirow{2}{*}{ B.O.D. (mg/L) } & $0.2689 \pm .1253^{\mathrm{a}}$ & $0.4752 \pm 0.1944^{\mathrm{b}}$ & $0.9181 \pm 0.3866^{\mathrm{c}}$ & $0.5485 \pm 0.3554^{\mathrm{b}}$ \\
\hline & $0.05-0.54$ & $0.07-0.72$ & $0.08-1.34$ & $0.07-1.240$ \\
\hline \multirow{2}{*}{$\mathrm{Cl}^{-}(\mathrm{mg} / \mathrm{L})$} & $7.9559 \pm .4497^{\mathrm{b}}$ & $7.7944 \pm 0.8796^{b}$ & $5.5200 \pm 1.4622^{\mathrm{a}}$ & $7.3796 \pm 0.9445^{b}$ \\
\hline & $6.70-8.34$ & $5.66-9.92$ & $2.44-7.68$ & $4.88-8.730$ \\
\hline \multirow{2}{*}{$\mathrm{PO}_{4}(\mathrm{mg} / \mathrm{L})$} & $0.0080 \pm .0048^{\mathrm{a}}$ & $0.0122 \pm 0.0051^{\mathrm{ab}}$ & $0.0182 \pm 0.0192^{b c}$ & $0.0253 \pm 0.0163^{c}$ \\
\hline & $0.00-0.02$ & $0.00-0.02$ & $0.00-0.08$ & $0.00-0.050$ \\
\hline \multirow{2}{*}{$\mathrm{SO} 4^{2-}(\mathrm{mg} / \mathrm{L})$} & $8.0459 \pm 4.2572^{\mathrm{a}}$ & $42.8696 \pm 20.7008^{c}$ & $52.5481 \pm 22.5888^{\mathrm{c}}$ & $25.3037 \pm 22.3564^{\mathrm{b}}$ \\
\hline & $1.74-16.07$ & $2.20-65.19$ & $3.18-76.91$ & $1.86-68.890$ \\
\hline \multirow{2}{*}{$\mathrm{S}_{2}^{-}(\mathrm{mg} / \mathrm{L})$} & $0.9274 \pm .5276^{\mathrm{a}}$ & $1.7267 \pm 0.8075^{\mathrm{b}}$ & $2.7089 \pm 0.9969^{b}$ & $1.6941 \pm 0.86461^{\mathrm{c}}$ \\
\hline & $0.13-1.88$ & $0.23-2.98$ & $0.73-4.28$ & $0.33-3.380$ \\
\hline \multirow{2}{*}{$\mathrm{Na}(\mathrm{mg} / \mathrm{L})$} & $29.6111 \pm 5.7599^{\mathrm{a}}$ & $41.4867 \pm 10.4312^{\mathrm{b}}$ & $28.0130 \pm 4.9064^{\mathrm{a}}$ & $33.9252 \pm 14.0934^{\mathrm{a}}$ \\
\hline & $18.90-42.20$ & $18.08-55.44$ & $18.78-37.08$ & $18.96-96.800$ \\
\hline \multirow{2}{*}{$\mathrm{K}(\mathrm{mg} / \mathrm{L})$} & $3.9630 \pm 0.9494^{\mathrm{ab}}$ & $4.3181 \pm 1.0136^{\mathrm{b}}$ & $3.7748 \pm 0.8917^{\mathrm{ab}}$ & $3.6252 \pm 0.8560^{\mathrm{a}}$ \\
\hline & $2.52-5.88$ & $2.64-6.08$ & $2.45-5.66$ & $2.56-5.440$ \\
\hline \multirow{2}{*}{ T.Hard.(mg/L) } & $128.6622 \pm 3.2795^{\mathrm{a}}$ & $159.5241 \pm 12.8838^{c}$ & $140.6163 \pm 8.5272^{b}$ & $143.1304 \pm 9.9597^{\mathrm{b}}$ \\
\hline & $123.08-134.90$ & $131.42-178.58$ & $127.42-154.46$ & $126.76-161.86$ \\
\hline \multirow{2}{*}{ T.Alka. (mg/L) } & $132.3330 \pm 2.9881^{\mathrm{a}}$ & $163.3478 \pm 13.0315^{\mathrm{c}}$ & $143.1726 \pm 7.9039^{\mathrm{b}}$ & $146.5430 \pm 9.4916^{\mathrm{b}}$ \\
\hline & $127.79-138.56$ & $136.02-183.38$ & $131.72-159.06$ & $130.32-162.420$ \\
\hline \multirow{2}{*}{$\operatorname{Mg}(\mathrm{mg} / \mathrm{L})$} & $7.4904 \pm 0.1395^{\mathrm{ab}}$ & $8.0778 \pm 0.4436^{\mathrm{c}}$ & $7.2581 \pm 0.78398^{\mathrm{a}}$ & $7.6589 \pm 0.1982^{b}$ \\
\hline & $7.20-7.79$ & $7.36-8.96$ & $5.53-8.08$ & $7.28-8120$ \\
\hline \multirow{2}{*}{$\mathrm{Ca}(\mathrm{mg} / \mathrm{L})$} & $7.9185 \pm 0.3265^{\mathrm{a}}$ & $8.7607 \pm 0.6639^{\mathrm{a}}$ & $8.0807 \pm 0.3650^{\mathrm{a}}$ & $10.6585 \pm 13.1236^{\mathrm{a}}$ \\
\hline & $7.43-8.63$ & $7.65-9.98$ & $7.56-8.89$ & $7.55-76.300$ \\
\hline & $0.0005 \pm 0.0003^{\mathrm{a}}$ & $0.0010 \pm 0.0004^{\mathrm{b}}$ & $0.0015 \pm 0.0005^{\mathrm{a}}$ & $0.0008 \pm 0.0004^{\mathrm{b}}$ \\
\hline $\mathrm{NO}_{2}(\mathrm{mg} / \mathrm{L})$ & $0.0001-0.0012$ & $0.0003-0.0019$ & $0.0003-0.0023$ & $0.0001-0.0017$ \\
\hline & $0.7519 \pm 0.3179^{\mathrm{a}}$ & $1.4444 \pm 0.6079^{b}$ & $2.7074 \pm 0.9148^{\mathrm{b}}$ & $1.5481 \pm 0.7387^{\mathrm{c}}$ \\
\hline $\mathrm{NO}_{3}(\mathrm{mg} / \mathrm{L})$ & $0.2000-1.4000$ & $0.3000-2.6000$ & $1.000-4.2000$ & $0.300-3.000$ \\
\hline $\mathrm{NH} / \mathrm{N}(\mathrm{mo} / \mathrm{I})$ & $0.0007 \pm 0.0004^{\mathrm{a}}$ & $0.0012 \pm 0.0006^{\mathrm{b}}$ & $0.0016 \pm 0.0005^{\mathrm{c}}$ & $0.0009 \pm 0.0004^{\mathrm{ab}}$ \\
\hline $\mathrm{NH}_{3}-\mathrm{N}$ & $0.0001-0.0015$ & $0.0002-0.0024$ & $0.0004-0.0026$ & $0.0001-0.0017$ \\
\hline $\mathrm{Fe}^{+}$ & $0.0043 \pm 0.0022^{\mathrm{a}}$ & $0.0093 \pm 0.0051^{\mathrm{b}}$ & $0.0039 \pm 0.0019^{\mathrm{a}}$ & $0.0057 \pm 0.0029^{a}$ \\
\hline $\mathrm{Fe}_{2}(\mathrm{mg} / \mathrm{L})$ & $0.0005-0.0090$ & $0.0010-0.0200$ & $0.001-0.009$ & $0.001-0.013$ \\
\hline & $0.9407 \pm 0.4299^{\mathrm{a}}$ & $1.3778 \pm 0.6880^{b}$ & $0.9704 \pm 0.4738^{a}$ & $0.6511 \pm 0.3524^{\mathrm{a}}$ \\
\hline $\mathrm{Fe}(\mu \mathrm{g} / \mathrm{L})$ & $0.100-1.800$ & $0.100-2.700$ & $0.100-2.000$ & $0.010-1.500$ \\
\hline & $13.7037 \pm 6.0308^{\mathrm{ab}}$ & $18.3704 \pm 8.8106^{\mathrm{b}}$ & $9.6667 \pm 4.7878^{\mathrm{a}}$ & $13.8148 \pm 7.2485^{\mathrm{ab}}$ \\
\hline $\mathrm{Cu}(\mu \mathrm{g} / \mathrm{L})$ & $1.00-25.000$ & $1.000-36.000$ & $1.000-21.00$ & $1.000-30.000$ \\
\hline $\mathrm{Cd}(\mathrm{u} / \mathrm{I})$ & $0.3481 \pm 0.2502^{\mathrm{a}}$ & $0.3111 \pm 0.2063^{\mathrm{a}}$ & $0.4963 \pm 0.4459^{\mathrm{ab}}$ & $0.6926 \pm 0.4787^{\mathrm{b}}$ \\
\hline $\mathrm{Cd}(\mu \mathrm{g} / \mathrm{L})$ & $0.00-0.900$ & $0.000-0.7000$ & $0.000-1.50$ & $0.000-1.700$ \\
\hline & $0.0027 \pm 0.0018^{\mathrm{a}}$ & $0.0051 \pm 0.003^{\mathrm{b}}$ & $0.0019 \pm 0.0013^{\mathrm{a}}$ & $0.0048 \pm 0.0044^{\mathrm{b}}$ \\
\hline $\mathrm{Hg}(\mu \mathrm{g} / \mathrm{L})$ & $0.00-0.0060$ & $0.000-0.010$ & $0.000-0.005$ & $0.000-0.016$ \\
\hline & $4.1111 \pm 2.2927^{\mathrm{ab}}$ & $5.7037 \pm 2.8932^{b}$ & $3.2963 \pm 1.8148^{\mathrm{a}}$ & $3.2963 \pm 1.8359^{\mathrm{a}}$ \\
\hline $\mathrm{Ni}(\mu \mathrm{g} / \mathrm{L})$ & $1.00-9.00$ & $1.000-11.000$ & $1.000-8.00$ & $1000-8.000$ \\
\hline & $6.1111 \pm 3.4344^{\mathrm{a}}$ & $11.7037 \pm 5.3192^{\mathrm{b}}$ & $5.7778 \pm 2.9000^{\mathrm{a}}$ & $5.8148 \pm 3.3859^{\mathrm{a}}$ \\
\hline $\mathrm{Zn}(\mu \mathrm{g} / \mathrm{L})$ & $1.000-13.000$ & $1.000-23.000$ & $1.000-12.00$ & $1.000-15.000$ \\
\hline
\end{tabular}


The total alkalinity and total hardness values in lime soils are generally close to each other (Boyd and Tucker, 2012). The alkalinity level of the natural waters varies between 5 and $500 \mathrm{mg} / \mathrm{L}$, and is closely related with the structure. The carbonate and bicarbonate give the alkalinity to the water (Tepe et al., 2006). According to the results obtained from our analyses, the brook shows mildly-hard water characteristics.

Among natural anions of the water, the presence of the sulfate $\left(\mathrm{SO}_{4}\right)$ in natural resources is important for improved biological productivity (Taş et al., 2010). The maximum limit for sulfate in water from the aspect of aquatic products was determined as $90 \mathrm{mg} / \mathrm{L}$ (Küçük, 2007). The maximum mean value was observed to be $45.24 \mathrm{mg} / \mathrm{L}$. Besides the sulfate, the chloride level is also an important parameter indicating the healthy water. The maximum mean chloride level of the brook was found to be $7.59 \mathrm{mg} / \mathrm{L}$ in fist station (Table $1 \mathrm{a}, \mathrm{b})$. These values are suitable for aquaculture.

The phosphor found in water resources is an element that is necessary of eutrophication (Haper, 1992). The reason of fluctuation in phosphorus level is the use of agricultural fertilizers containing phosphor, because there are many farms near the brook. The maximum mean level of phosphate in the brook was found to be $0.03 \mathrm{mg} / \mathrm{L}$ in $9^{\text {th }}$ station (Table 1a,b). This phosphate concentration poses no danger in terms of aquaculture and aquatic life.

$\mathrm{Ca}^{++}$and $\mathrm{Mg}^{++}$are the most important dissolved solid matters in water (Mutlu et al., 2013b). $\mathrm{Mg}^{++}$and $\mathrm{Ca}^{++}$are alkali soil minerals, and are among the ions existing in fresh waters at most. The maximum recommended $\mathrm{Ca}^{++}$ level is reported to be $75 \mathrm{mg} / \mathrm{L}$ (Taş, 2006). In this study, the maximum calcium $\left(\mathrm{Ca}^{++}\right)$level was found to be 13.37 $\mathrm{mg} / \mathrm{L}$ in first station. This calcium concentration indicates that the amount of $\mathrm{Ca}^{++}$in Brook Kuruçay is within the acceptable limits (WHO, 2011).

The concentration of magnesium in normal waters should be between $5 \mathrm{mg} / \mathrm{L}$ and $60 \mathrm{mg} / \mathrm{L}$. In mildly hard waters, the values between 60 and $100 \mathrm{mg} / \mathrm{L}$ can be accepted as typical, and the recommended concentration of $\mathrm{Mg}^{++}$is $50 \mathrm{mg} / \mathrm{L}$ (Taş, 2006). In this study performed in Brook Kuruçay, the maximum mean value found is $7.88 \mathrm{mg} / \mathrm{L}$ in $8^{\text {th }}$ station (Table 1a,b).

The concentration of Potassium $(\mathrm{K})$ and Sodium $(\mathrm{Na})$ vary within the ranges of $1-10 \mathrm{mg} / \mathrm{L}$ and $2-100 \mathrm{mg} / \mathrm{L}$ in natural waters, respectively (Boyd, 1998). The maximum mean potassium concentration in our study was determined to be $5.54 \mathrm{mg} / \mathrm{L}$ that can be considered to be within the normal ranges, while the maximum level of sodium concentration was found to be $40.75 \mathrm{mg} / \mathrm{L}$ in $9^{\text {th }}$ station. Under the light of these results, it can be concluded that the sodium and potassium concentrations can be considered to be within the acceptable limits (WHO, 2011, 2015).

The waters having lead $(\mathrm{Pb})$ concentration of 0.01 $\mathrm{mg} / \mathrm{L}$ and higher are considered to be polluted. It has also been reported that the lead affects the osmotic balance and ion arrangement in fish and leads to histopathologic change in liver (Atay and Pulatsü, 2000). Although the presence of cadmium $(\mathrm{Cd})$ in waters at the concentration of $5 \mu \mathrm{g} / \mathrm{L}$ and higher is reported to be toxic and it directly leads to mortality in aquatic organisms at high concentrations, it also leads to metabolic and physiologic disorders and changes especially in fish (Mutlu et al., 2013c). The maximum concentrations of lead $(\mathrm{Pb})$ and cadmium (Cd) in Brook Kuruçay were calculated to be $1.817 \mu \mathrm{g} / \mathrm{L}$ and $0.833 \mu \mathrm{g} / \mathrm{L}$, respectively. The reason of this this level of cadmium concentration in the brook can be attributed to the artificial phosphate fertilizers used for the agricultural activities around the lake. Under the lights of those values, it was determined that the brook shows Class I water characteristic in terms of lead $(\mathrm{Pb})$ and cadmium (Cd) elements according to RSWQM.

Maximum mean concentration of the copper $(\mathrm{Cu})$ element was found to be $25.167 \mu \mathrm{g} / \mathrm{L}$ in $9^{\text {th }}$ station (Table $1 \mathrm{a}, \mathrm{b})$. The reason of this concentration is thought to be caused from the penetration of copper, which was accumulated in the soil due to dense usage of copper vitriol during maintenance and pruning processes in fruit gardens in spring season, into the brook waters through rain. According to the RSWQM, the brook shows Class I water characteristic in terms of copper $(\mathrm{Cu})$.

The maximum mean concentration of ferrous $\left(\mathrm{Fe}^{2+}\right)$ in the brook was found to be $0.011 \mathrm{mg} / \mathrm{L}$ in first station (Table 1a,b). The concentrations of ferrous have peaked during summer season due to wide wheat planting around the lake. Since the use of ferrous-containing agricultural pesticides in order to increase the grain productivity of wheat plants increases especially between May and June, the ferrous-containing waters and particles may leak into the brook through rain and leakages.

The maximum mean concentrations of Zinc ( $\mathrm{Zn})$, nickel (Ni) and mercury $(\mathrm{Hg})$ during the study were found to be $9.00 \mu \mathrm{g} / \mathrm{L}, 7.75 \mu \mathrm{g} / \mathrm{L}$, and $0.007 \mu \mathrm{g} / \mathrm{L}$, respectively (Table 1a,b). This level of mercury concentration in the brook may be caused by flows from cultivation areas into the lake, since the use of fertilizers is very common in Hafik district. The level of concentration of zinc is caused by the incineration of wastes of mining and coal mining industries and the processes of iron and steel industry. It is used as oxide stain material in plastics, cosmetics, copy and wall papers, printer inks, ceramics, rubber industry, and fertilizers. Under the lights of these values, it was determined that the brook shows Class I water characteristic in terms of zinc $(\mathrm{Zn})$, mercury $(\mathrm{Hg})$, and nickel (Ni) according to RSWQM.

\section{Conclusion}

It is known that the heavy metals constitute an important pollutant group, and they incline to accumulate within the bodies of living organisms, as well as they have significant toxic and carcinogenic effects. Heavy metals having strong poisonous effects even at very low concentrations may inhibit the self-cleaning process of natural waters, and they also affect water sources' usability in irrigation and aquaculture negatively. As it can be seen in results of the analyses, the water quality in Brook Kuruçay is considered to be Class I according to RSWQM. In order to protect the water quality and to ensure the health of aquatic life in this brook, it is required to make regular observations and to monitor the parameters affecting the water quality and aquatic life.

\section{References}

Acu A. 2000. Beytepe Göleti' nin Su Kalitesinin Belirlenmesi Üzerine Bir Araştırma. Postgraduate Thesis, Department of Aquaculture, Science Institute of Ankara University, Ankara- Türkiye. 
Akkan T, Kaya A, Dinçer S. 2011. Hastane Atık Sularıyla Kontamine Edilen Deniz Suyundan İzole Edilen Gram Negatif Bakterilerin Sefalosporin Grubu Antibiyotiklere Karşı Direnç Düzeyleri, Türk Mikrobiyoloji Cemiyeti Dergisi, 4(1): 18-21.

Alaş A, Çil OŞ. 2002. Aksaray İline İçme Suyu Sağlayan Bazı Kaynaklarda $\mathrm{Su}$ Kalite Parametrelerinin İncelenmesi. Ekoloji Dergisi, 11(42): 40-44.

Anonymous. 1998. Standard Methods for the Examination of Water and Wastewater. Washington, DC: American Public Health Association; American Works Association; Water Environment Federation.

Atay D, Pulatsü S. 2000. Su Kirlenmesi ve Kontrolü. Ankara Üniversitesi Ziraat Fakültesi, Publication No: 1513, Ankara.

Boyd CE. 1998. Water Quality for Pond Aquaculture, Alabama Agricultural Experiment Station, Research and Development Series No: 43, Auburn- USA.

Boyd CE, Tucker CS. 2012. Pond Aquaculture Water Quality Management, Kluwer Academic Publishers,700.

Gleick PH. 1996. Basic Water Requirements for Human Activities: Meeting Basic Needs, Water International, 21, 83-92.

Güler Ç. 1997. Su Kalitesi. TR Ministry of Health, Çevre Sağlık Kaynak Dizisi, No:43, p: 92, Ankara.

Harper D. 1992. Eutrophication of Freshwaters: Principles, Problems and Restoration London, Chapyen Hall. 78.

İmamoğlu Ö. 2000. Dipsiz ve Çini (Muğla - Aydın) Çayı'nın Su Kalitesinin Fizikokimyasal ve Biyolojik (Bentik Makroinvertebrat) Yönden İncelenmesi. Postgraduate Thesis, Department of Biology, Science Institute of Muğla University, Muğla-Türkiye.

Kara C, Gömlekçioğlu V. 2004. Karaçay (Kahramanmaraş) 'ın Kirliliğinin Biyolojik ve Fiziko- Kimyasal Parametrelerle İncelenmesi. Kahramanmaraş. Sütçü İmam Üniversitesi Fen ve Mühendislik Dergisi 7(1): 1-7.

Kaptan H, Özan ST. 2014. Eğirdir Gölü'nün (Isparta) Suyunda, Sedimentinde ve Gölde Yaşayan Sazan' in (Cyprinus carpio L. 1758) Bazı Doku ve Organlarındaki Ağır Metal Düzeylerinin Belirlenmesi. S.D.U. Journal of Science, 9 (2): 44-60.

Kurnaz A, Mutlu E, Aydin Uncumusaoğlu A. 2016. Determination of Water Quality Parameters and Heavy Metal Content in Surface Water of Çiğdem Pond (Kastamonu/Turkey) Turkish Journal of Agriculture - Food Science and Technology, 4(10): 907-913.

Küçü, S. 2007. Investigation of Water of Quality Parameters of the Büyük Menderes River for Fisheries. A.D.U. Journal of the Faculty of Agriculture, 4(1-2),7-13.

Mutlu E, Yanik T, Demir T. 2013a. Karagöl (Hafik-Sivas)'ün $\mathrm{Su}$ Kalitesinin İncelenmesi, Alınteri Ziraat Bilimler Dergisi, 24, 35-45.
Mutlu E, Yanik T, Demir T. 2013b. Horohon Deresi (HafikSivas) Su Kalitesi Özelliklerinin Aylık Değişimleri. Alınteri Zirai Bilimler Dergisi, 25 (B), 45-57.

Mutlu E, Demir T. Kutlu B, Yanik T. 2013c. Sivas - Kurugöl Su Kalite Parametrelerinin Belirlenmesi. Türk Tarım-Gıda Bilim ve Teknoloji Dergisi 1(1): 37-43.

Mutlu E, Kutlu B, Yanik T, Demir T. 2014. Faraz Stream (Hafik - Sivas) Water Quality Characteristics and Monthly Variations. Standard Scientific Research and Essays, 2(11): 587-594.

Ntengue FW. 2006. Pollutant loads and water quality in streams of heavily populated and industrialized towns. Physics and Chemistry of the Earth, Parts A/B/C, 31(15-16): 832-839.

Polat N, Akkan T. 2016. Assessment of Heavy Metal and Detergent Pollution in Giresun Coastal Zone, Turkey. Fresenius Environmental Bulletin, 25(8): 2884-2890.

RSWQM. 2015. Regulation on the surface water quality management, number of official gazette: 29327.

Taş, B., 2006. Derbent Baraj Gölü (Samsun) Su Kalitesinin İncelenmesi. Ekoloji, 15, (61), 6-15.

Taş, B., Candan, A.Y., Can, Ö.V. and Topkara, S. 2010. Ulugöl (ORDU)'nun Bazı Fiziko-Kimyasal Özellikleri. Journal of Fisheries Sciences,4(3): 254-263.

Taş B. 2011. Gaga Gölü (Ordu,Türkiye) Su Kalitesinin İncelenmesi. Black Sea Technical University's Journal of Science, 1(3): 43-61.

Tepe Y, Ateş A, Mutlu E, Töre Y. 2006. Karagöl'ün (ErzinHatay) Bazı Fiziko-Kimyasal Özellikleri”, Ege University Journal of Fisheries, 23(1/1): 155-161.

Topal M, Arslan Topal E. 2012. Elazı̆ ilinde Bir Maden Sahasında Kaynaklanan Sızıntı Sularının Maden Çayına Etkisi. Karaelmas Üniversitesi Fen ve Mühendislik Dergisi, 2(1): 15-21, Zonguldak.

Tüzün İ, İnce Ö, Başaran G. 2006. Doğal Göl ve Rezervuar Limnolojisindeki Farklılıkların Birleşik Yönetim Planlanması Açısından Değerlendirilmesi, 1. Balıklandırma ve Rezervuar Yönetimi Sempozyumu 07-09 Şubat 2006, Antalya.

Verep B, Serdar U, Turan D, Şahin C. 2005. İyidere (Trabzon)'nin Fiziko-Kimyasal Açıdan $\mathrm{Su}$ Kalitesinin Belirlenmesi. Ekoloji, 14(57): 26-35.

WHO. 2011. World Health Organization, Guidelines for drinking-water, Geneva Switzerland. 4th ed. ISBN 9789241548151.

WHO. 2015. World Health Organization. Sodium in Drinkingwater, Guidelines for Drinking-water Quality, Health criteria and other supporting information, Geneva Switzerland, 2nd ed. (2). 